\title{
Mesure en continu des matières en suspension à l'aide d'un photomètre à fibre optique
}

\author{
Continuous measurement of suspended solids \\ with an optical fiber photometer
}

\author{
A. Paitry \\ Service départemental d'assainissement \\ Rosny-sous-Bois
}

Afin de gérer les ouvrages d'assainissement à partir de critères qualitatifs, outre les critères hydrauliques, les techniques du Service d'assainissement de la Seine Saint-Denis ont expérimenté un spectrophotomètre qui a pour originalité d'utiliser une fibre optique.

La granulométrie des matériaux, le choix de la longueur d'onde, les interférences des substances colorées, l'encrassement de la cellule de mesure ont fait l'objet d'essais préliminaires.

Des corrélations entre la mesure de MES AFNOR et la densité optique ont été établies pour cinq collecteurs par temps sec et lors d'événements pluvieux. Les résultats sont très intéressants : pour les 374 échantillons concernés, le coefficient de corrélation est compris entre 0,906 et 0,961.

Ce matériel est maintenant installé à demeure sur un collecteur pluvial.

In order to manage sewage with quality informations in addition to hydraulic data, the Seine Saint-Denis county technicians experimented a typical photometer characterized by the use of an optic fiber.

Suspended solids size, choice of the wave length, effects of colored substances, and sedimentation in the measurement cell were preliminary tested.

Correlations between the suspended solids measures by AFNOR method and by optic measure, 374 samples were collected, were established on waste waters and runoff waters of five sewers; results are very interesting; $\mathrm{Cr}$ is included between 0,906 and 0,961.

This apparatus is now working at full time on a storm sewer.

En période de forte pluie, la gestion de certains réseaux d'assainissement des agglomérations peut être rendue difficile. Les gestionnaires sont en effet parfois confrontés à deux exigences contradictoires : celle d'éviter l'inondation des points bas des zones de collecte et des habitations qui s'y trouvent et celle d'éviter la pollution du milieu récepteur.

Par temps de pluie la gestion des eaux transportées par les collecteurs passe, non seulement par une bonne connaissance des débits, mais aussi par la connaissance des données qualitatives et ceci en temps réel : on peut par exemple, envisager d'envoyer vers une station d'épuration de capacité insuffisante les eaux les plus chargées, après mesure comparative des qualités des eaux des divers collecteurs affluents, et rejetter les eaux les moins chargées au milieu naturel. Autre exemple, celui des eaux de ruissellement dans les réseaux séparatifs, qui, lorsqu'elles atteignent une trop mauvaise qualité, pourraient être stockées dans des bassins placés en dérivation avant renvoi sur la station.

LA HOUILLE BLANCHE/N ${ }^{\circ}$ 3-1987 


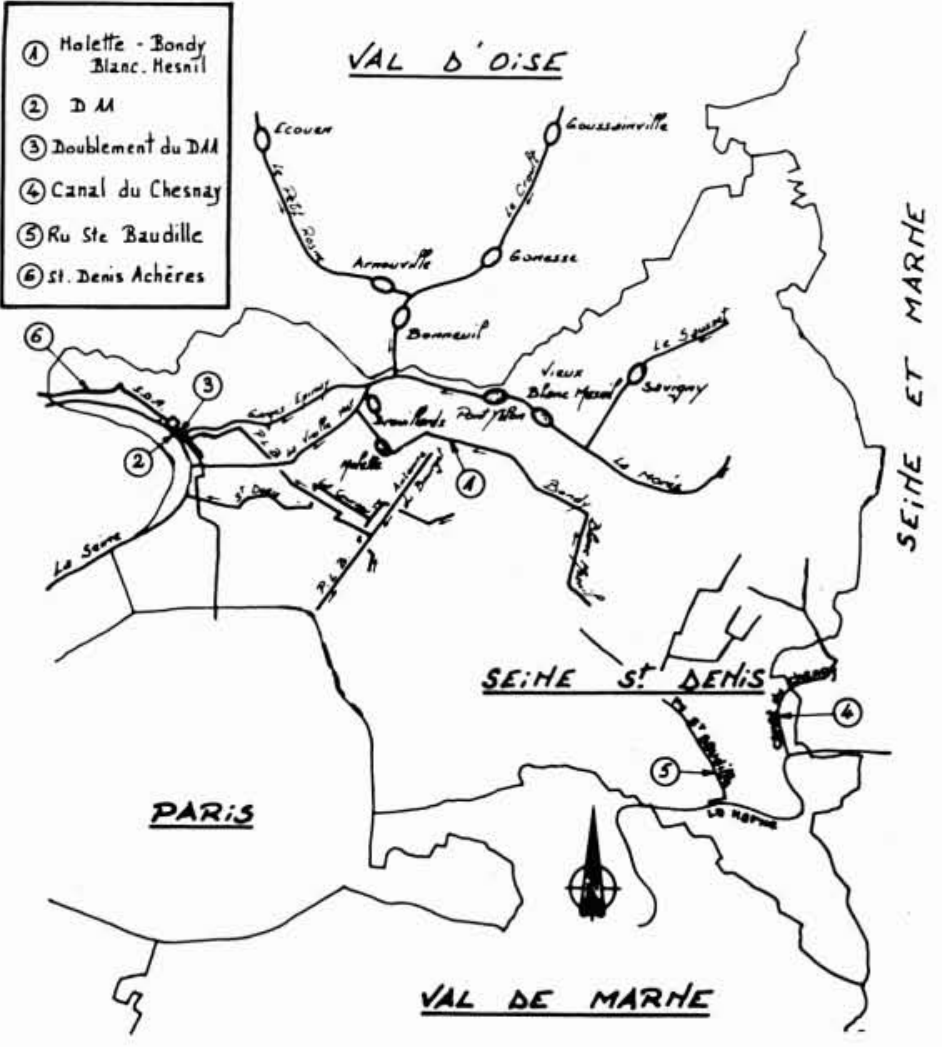

1 .

La mesure instantanée et en continu de la qualité des effluents domestiques et pluviaux constitue donc une étape indispensable dans l'amélioration de la gestion des écoulements. Dans ce contexte, le Département de SeineSaint-Denis a développé depuis plusieurs années un programme d'études destinées à maîtriser la gestion de ses réseaux d'assainissement et à permettre à terme de diminuer sensiblement les flux de pollution envoyés au milieu naturel.

Parmi ces études, les recherches sur les mesures de qualité se sont tournées vers la connaissance des matières en suspension, paramètre le plus facilement accessible pour une mesure en continu. Des automates sont en effet capables de reproduire fidèlement les conditions définies par certaines normes d'analyses chimiques de laboratoire (DCO-mètre par exemple). Mais leurs conditions de mise en œuvre sont tout à fait incompatibles avec les contraintes qui prévalent sur les sites de mesure. Parmi les caractéristiques que doit présenter un appareillage de mesure sur le terrain, il faut citer en effet la facilité d'emploi, la résistance à l'humidité, des réactifs bon marché nécessaires en petite quantité, ou mieux encore l'absence totale de réaction, la fiabilité et la représentativité. Par ailleurs, si certaines déterminations sont réalisables automatiquement, le résultat de la mesure n'est pas toujours instantané (notamment dans le cas d'analyses chimiques).

De nombreuses expérimentations ont été réalisées par divers organismes pour relier la turbidité à la mesure des MES (telles que déterminées selon la norme AFNOR*).
Ces expérimentations se sont souvent heurtées à des problèmes de fiabilité du matériel ou d'encrassement des capteurs.

C'est l'apparition du photomètre à fibre optique qui nous a permis de poursuivre cette démarche avec du matériel a priori mieux adapté.

Les échantillons d'eaux qui ont fait l'objet de mesures simultanées par la méthode AFNOR et à l'aide du spectrophotomètre proviennent de sites sur lesquels il existe des possibilités de gestion faisant appel à la qualité de l'effluent.

Les échantillonnages ont été réalisés dans des collecteurs pluviaux de réseaux séparatifs et pseudo-séparatifs (Canal du Chesnay et Ru St. Baudille), dans un émissaire pluvial (Bondy-Blanc-Mesnil) et dans des collecteurs unitaires (D11, son doublement et Saint-Denis-Achères). Une description rapide de ces collecteurs et de leurs bassins versants respectifs est faite au chapitre 3.1.

Depuis l'été 1985 le spectrophotomètre géré par un microprocesseur est installé à demeure sur le terrain pour effectuer des mesures comparatives évoquées ci-avant sur les eaux de la Vieille Mer. Ce très gros collecteur pluvial du Nord du Département alimente soit un bassin de rétention $\left(140000 \mathrm{~m}^{3}\right)$, soit le Garges Epinay, émissaire pluvial.

\section{Détermination du plan d'expérience}

\subsection{Le choix de l'indicateur de qualite}

Les deux principaux critères qui ont guidé notre choix sont :

- la possibilité d'accéder à une grandeur mesurable de manière simple corrélable avec l'un des indicateurs de pollution couramment utilisés dans le domaine de l'assainissement et,

- que cet indicateur donne une image représentative de la qualité de l'effluent.

Les déterminations les plus souvent réalisées sont les suivantes :

DCO, $\mathrm{DBO}_{s}, \mathrm{MES}, \mathrm{NH}_{4}^{+}, \mathrm{NTK}, \mathrm{N}$ Total, $\mathrm{Pb}, \mathrm{Zn}, \mathrm{Cd}, \mathrm{Hg}$, $\mathrm{Hc}$

Les trois premiers indicateurs sont couramment utilisés pour caractériser la qualité des eaux domestiques ou pluviales. Dans le cas d'effluents industriels les indicateurs choisis sont fonction de l'activité. Le choix des MES comme indicateur de qualité nous a semblé répondre à ces deux critères.

\subsection{Le choix de la mesure}

Il n'était pas question de reproduire la mesure réalisée dans le cadre de la Norme, mais d'effectuer une mesure simple corrélable avec la mesure de MES.

De nombreuses mesures réalisées à l'aide de spectrophotomètres dans le visible ont montré que malgré les

* Voir bibliographie 
limites d'une telle mesure, on pouvait espérer des informations intéressantes. L'utilisation du pulvérimètre à laser a été envisagée mais cet appareil était difficilement utilisable sur le terrain dans sa version d'origine. De plus il n'avait pas été testé pour des particules de quelques dizaines à plusieurs centaines de microns.

Sur la base de travaux antérieurs, notamment ceux de Ben Aim et Coll. nous avons retenu une mesure spectrophotométrique d'absorbance à $510 \mathrm{~nm}$.

A concentration égale en MES, l'absorbance apparente varie selon la granulométrie des particules, celles de plus grande taille conduisant à l'absorbance spécifique la plus faible. Dans ces conditions, et quel que soit l'étalon utilisé, les possibilités d'obtention d'une relation Absorbance $=$ $\mathrm{f}(\mathrm{MES})$ unique apparaissent des plus restreintes.

Par contre, en un point de mesure donné d'un collecteur et sous réserve de réaliser un étalonnage comparatif Absorbance/MES AFNOR intégrant un nombre suffisant d'événements pluvieux il nous a semblé possible d'obtenir une approximation satisfaisante du taux de MES.

\subsection{Vérification de l'hypothèse de travail}

\subsubsection{L'émissaire pluvial Bondy Blanc-Mesnil :}

Il est alimenté par des surverses des réseaux unitaires. Les eaux de ruissellement échantillonnées correspondent à une exception près à des événements pluvieux de faible importance pour lesquels la dilution des eaux usées était donc faible. 91 mesures ont été réalisées à l'aide du spectrophotomètre IL-253 avec cuves à circulation en quartz.

\subsubsection{Résultats}

Des essais de vérification des hypothèses de travail ont été menés avec un spectrophotomètre à double faisceau équipé d'une cellule à circulation en quartz de $1 \mathrm{~cm}$ de chemin optique, d'une lampe à cathode creuse au tungstène, d'une pompe de circulation et d'un enregistreur potentiométrique. Une première série d'essais menée sur des suspensions de Kaolin a permis de déterminer le domaine de linéarité de la réponse.

Pour chaque crue on obtient une très bonne corrélation entre l'absorbance et les MES AFNOR. Par contre les pentes des droites en régression varient du simple au double, en particulier en fonction du débit maximum.

Lorsque l'on regroupe l'ensemble des mesures, les teneurs déduites de la droite de régression moyenne se situent dans $80 \%$ des cas à moins de $25 \%$ de la teneur déterminée par la méthode AFNOR. Cette différence intègre celle propre à l'évaluation des MES.

\subsection{Description de l'appareil de mesure}

La plupart des photomètres ou turbidimètres commerciaux, conçus pour des usages de laboratoire, sont - le plus souvent - peu adaptés aux mesures sur le terrain.

L'utilisation de fibres optiques permet de séparer l'analyseur de la cellule de mesure et de concevoir des cellules ouvertes moins susceptibles de s'encrasser ou de se détériorer.
La société Métrohm propose à un prix modéré un photomètre très simple équipé d'une fibre optique.

La lumière issue d'une lampe halogène de $10 \mathrm{~W}$ traverse un filtre dégradé qui permet (selon le constructeur) de fixer la longueur d'onde entre 420 et $700 \mathrm{~nm}$ avec une résolution de $10 \mathrm{~nm}$. La lumière est ensuite séparée en deux faisceaux modulés optiquement à deux fréquences distinctes grâce à un obturateur tournant.

Le faisceau lumineux de référence parvient directement au détecteur, le faisceau de mesure emprunte la fibre aller, traverse l'échantillon d'effluent, est réfléchi sur un miroir concave et guidé par la fibre optique retour au photodétecteur.

Le système fonctionne donc en double faisceau. La lumière incidente sensiblement monochromatique permet de fixer la longueur d'onde sans avoir recours à une gamme de filtres interférentiels.

Les fibres optiques aller et retour de section semicirculaire sont accolées et réunies en un guide unique qui aboutit à la cellule de mesure. Celle-ci est constituée d'un étrier en acier inoxydable supportant le miroir concave. On a donc une cellule de mesure ouverte. Son chemin optique est de $20 \mathrm{~mm}$.

L'affichage numérique fournit soit une mesure d'absorbance, soit une mesure de transmission. Le signal numérique n'est ni amorti, ni intégré.

La rusticité et la simplicité d'emploi de ce photomètre bien que tout à fait souhaitables pour des mesures de terrain sont toutefois assorties de quelques inconvénients notamment, l'existence d'une fibre optique trop courte, l'absence de dispositifs d'intégration du signal, de zéro automatique ou de sortie analogique proportionnelle à l'absorbance.

Un nouveau modèle (METROHM 662) commercialisé depuis début 1985 et fondé sur le même principe est en court d'évaluation et semble comporter quelques améliorations.

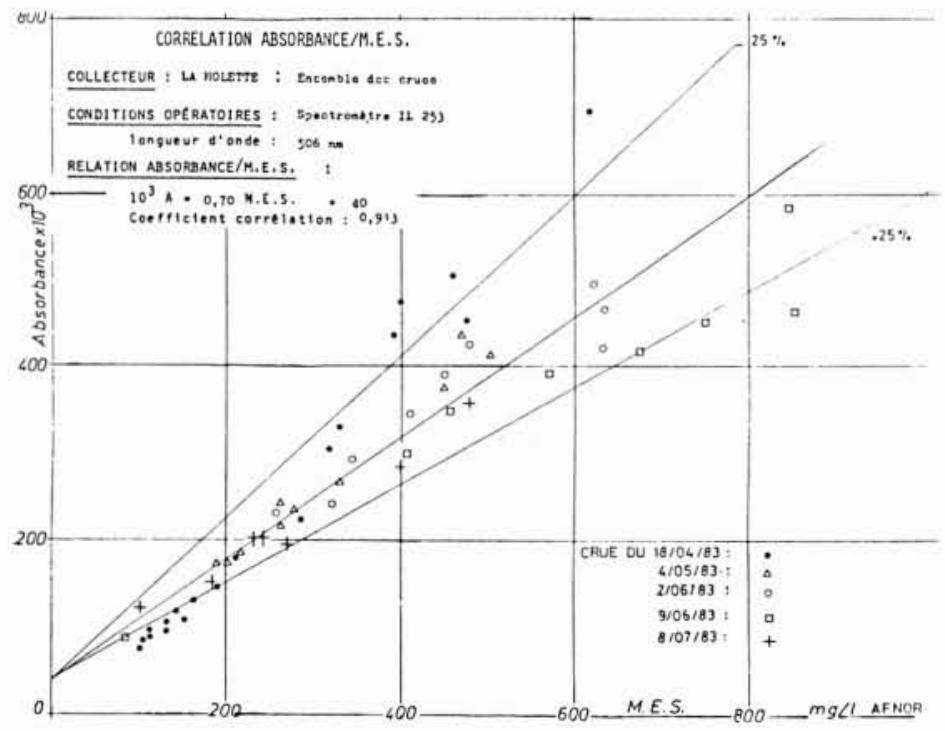

2. 


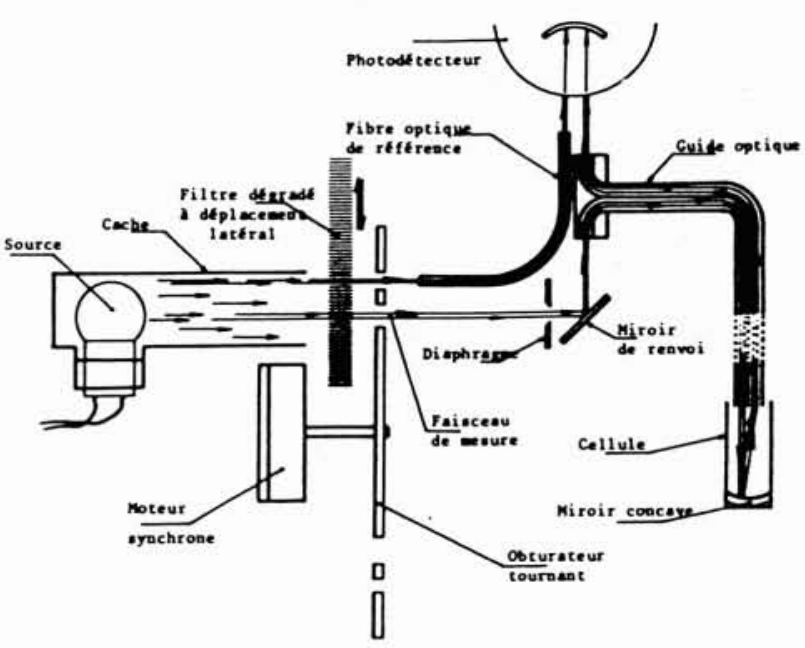

3. Schéma optique de principe.

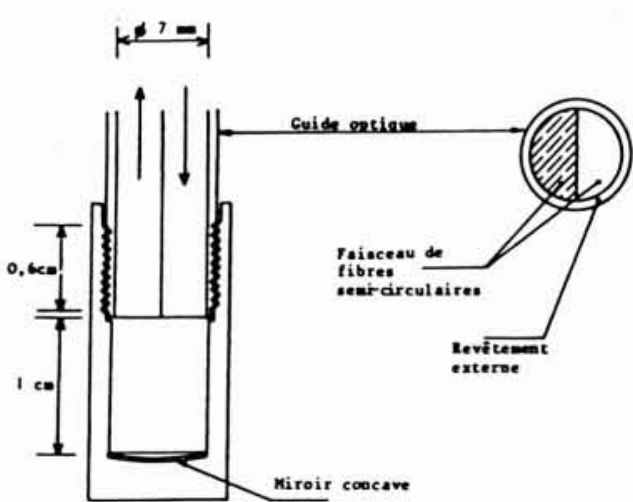

4. Coupe de l'extrémité du guide optique.

\section{Aptitude du photomètre à fibre optique à répondre aux objectifs assignés}

\subsection{Influence de la longueur d'onde}

Dans le cadre des travaux antérieurs et notamment ceux de Ben Aim et Coll., la longueur d'onde choisie pour soustraire l'absorbance propre aux MES était une mesure à $510 \mathrm{~nm}$ qui a été retenue pour la suite des expérimentations car entre 450 et $550 \mathrm{~nm}$ la variation d'absorbance d'une solution de kaolin est de $15 \%$.

\subsection{Interférences des substances colorées minérales et organiques}

Ces essais ont été réalisés pour évaluer les interférences éventuelles de substances colorées susceptibles d'être rencontrées dans les effluents industriels. Les essais ont porté sur des solutions d'ions minéraux colorés.

On observe des absorbances nulles pour des solutions à

$1 \mathrm{~g} / 1$ de Cuivre $\left(\mathrm{Cu}^{++}\right)$, Manganèse $\left(\mathrm{N}^{++}\right)$, Nickel $\left(\mathrm{Ni}^{++}\right)$, $0,3 \mathrm{~g} / \mathrm{l}$ de Vanadium $\left(\mathrm{VO}_{3}\right)$

$0,1 \mathrm{~g} / 1$ de Cobalt $\left(\mathrm{Co}^{++}\right)$ou Fer $\left(\mathrm{Fe}^{+++}\right.$ou $\left.\mathrm{Fe}^{++}\right)$.

Les composés du chrome $\left(\mathrm{Cr}^{\mathrm{II \prime}}\right.$ ou $\left.\mathrm{Cr}^{\mathrm{vl}}\right)$ n'induisant des interférences appréciables que pour des teneurs supérieures au $\mathrm{mg} / \mathrm{l}$. (Teneurs que l'on ne rencontre qu'en cas de pollution accidentelle). Le permanganate absorbe fortement à $510 \mathrm{~nm}$ mais sa présence dans des effluents est tout à fait improbable.

\subsection{Encrassement de la cellule}

Aucun encrassement notable n'a été noté après un fonctionnement continu durant 15 heures.

\subsection{Influence de la granulométrie}

Pour apprécier l'influence de la granulométrie des MES sur la réponse photométrique, des essais ont été réalisés sur des échantillons reconstitués à partir de fractions granulométriques connues de sédiments en provenance d'un bassin de rétention-décantation. On voit que les pentes des courbes peuvent alors être très différentes.

\subsection{Linéarité de la mesure}

Pour une suspension de kaolin, au-delà de $500 \mathrm{mg} / 1$ on note une distorsion de la linéarité du signal. L'extinction totale est obtenue pour une concentration de $1500 \mathrm{mg} / \mathrm{l}$.

\section{Mesures comparatives MES AFNOR. Absor- bance par photomètre à fibre optique sur des effluents}

Les mesures ont été réalisées sur des échantillons d'eau prélevés par un préleveur automatique et soumis successivement à une mesure photométrique et à une analyse de MES par la méthode AFNOR.

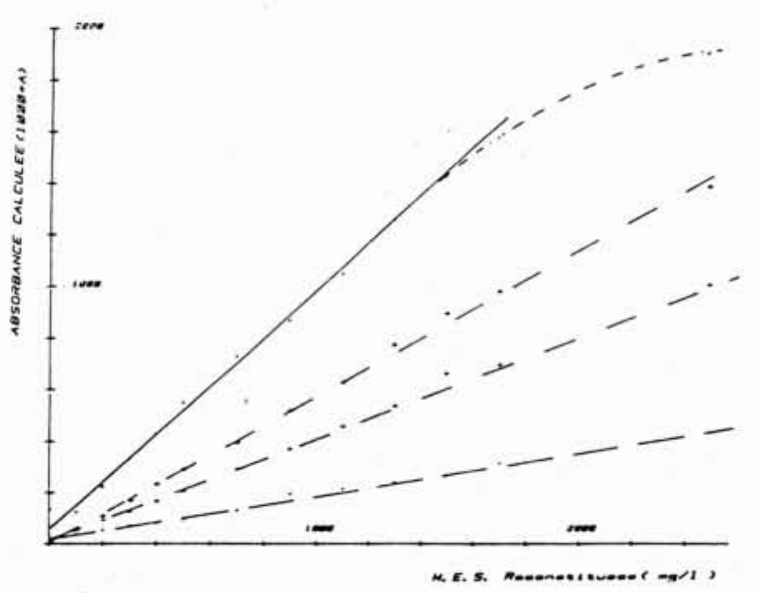

5. Influence de la granulométrie des MES. 
Tous ces essais ont été réalisés en laboratoire, leur but étant de vérifier la validité de la mesure et d'établir des courbes de correspondance MES AFNOR-MESURE optique. La mise en œuvre de l'appareil sur le terrain correspond à une phase ultérieure de cette expérimentation.

\subsection{Description des sites de prélèvement}

\subsubsection{Le collecteur unitaire D11 :}

Il amène des eaux en provenance pour l'essentiel des arrondissements Nord et Est de Paris. Les eaux de ruissellement échantillonnées correspondent donc à un mélange d'eaux usées et d'eaux pluviales. Le débit d'eaux usées peut varier de quelques centaines de litres par seconde à $1,5 \mathrm{~m}^{3} / \mathrm{s}$ en fonction de l'heure et de la gestion des collecteurs. La qualité des eaux échantillonnées est donc extrêmement variable. Les comparaisons ont porté sur 41 mesures.

Des échantillonnages ont également été réalisés sur les eaux unitaires par temps sec sur trois cycles de $24 \mathrm{~h}$ et ont conduit à 61 mesures comparatives ce qui porte le total des mesures à 102 .

\subsubsection{Le doublement du collecteur D11:}

Le DD 11 est également un collecteur de type unitaire qui transporte peu d'eaux usées. Les eaux de ruissellement échantillonnées correspondent à cinq événements pluvieux au cours desquels nous avons constitué 41 échantillons soumis aux mesures comparatives.

\subsubsection{L'émissaire St Denis Achères :}

Des prélèvements par temps sec ont également été réalisés sur l'émissaire St-Denis Achères qui transporte $5 \mathrm{~m}^{3} / \mathrm{s}$ d'un mélange d'eaux usées et d'eaux unitaires. Les échantillonnages réalisés sur 3 cycles de 24 heures ont conduit à 72 mesures comparatives.

\subsubsection{Canal du Chesnay}

Ce collecteur pluvial se jette en Marne en rive droite. Le collecteur transporte des eaux usées diluées dues à la mauvaise séparation des eaux. La partie avale du bassin versant est très plate à l'opposé de l'amont très pentu. Les mesures ont été réalisées sur les eaux de ruissellement.

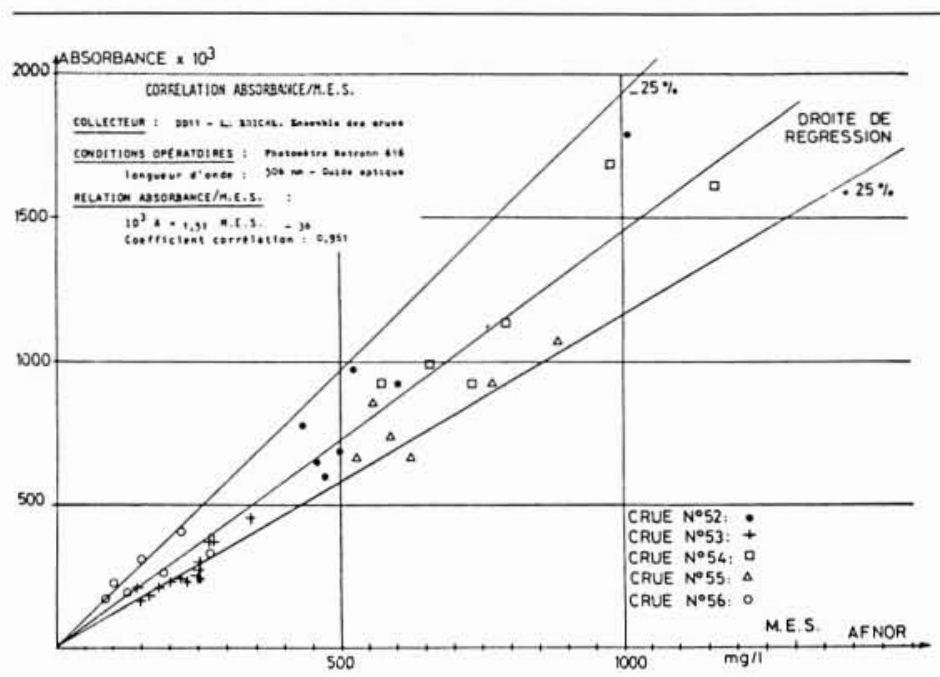

6.

\subsubsection{Ru St Baudille}

Ce collecteur pluvial transporte par temps sec 1201 /s d'un mélange d'eaux usées et d'eaux pluviales. Le débit de ruissellement peut atteindre $18 \mathrm{~m}^{3}$ et correspond à un mélange d'eaux pluviales et de surverses de réseaux unitaires. Le rejet se fait en rive droite de Marne et, comme pour le Canal du Chesnay, le bassin versant est pentu à l'amont et plat à l'aval.

\subsection{Les résultats obtenus}

Nous avons recherché des corrélations absorbance/MES. La droite obtenue sur le site du DD 11 est présentée sur la figure 6. $80 \%$ des valeurs mesurées se trouvent dans la limite de $\pm 25 \%$ des valeurs données par la droite de régression.

Le tableau suivant nous donne pour chaque collecteur étudié et pour l'ensemble des crues échantillonnées, l'équation des courbes de régression et le coefficient de corrélation. Pour le D11 et le ru St Baudille les corrélations du type $y=a x^{b}$ sensiblement plus favorables que les régressions linéaires ont été indiquées dans le tableau ci-après. Les tests de validité montrent que les deux variables ne sont pas indépendantes.

\begin{tabular}{|c|c|c|c|c|c|c|c|}
\hline Collecteur & $\begin{array}{c}\text { Nombre échan } \\
\text { tillons }\end{array}$ & & gr & $\begin{array}{l}\text { rbe de } \\
\text { ession }\end{array}$ & & $\begin{array}{l}\mathrm{Cc} \\
\mathrm{cor}\end{array}$ & $\begin{array}{l}\text { f. de } \\
\text { rélation }\end{array}$ \\
\hline D11 & $\begin{array}{l}102 \\
102\end{array}$ & $\begin{array}{l}10^{3} \mathrm{~A} \\
10^{3} \mathrm{~A}\end{array}$ & $\begin{array}{l}= \\
=\end{array}$ & $\begin{array}{l}0,930 \\
2,944\end{array}$ & $\begin{array}{l}\text { MES } \\
\times \quad(M E\end{array}$ & $+1,5,86$ & $\begin{array}{l}0,934 \\
0,951\end{array}$ \\
\hline DD 11 & 41 & $10^{3} \mathrm{~A}$ & $=$ & $1,51 \mathrm{M}$ & MES & 36 & 0,961 \\
\hline SDA & 72 & $10^{3} \mathrm{~A}$ & $=$ & 1.011 & MES & +126 & 0,906 \\
\hline Chesnay & 120 & $10^{3} \mathrm{~A}$ & $=$ & 1,251 & MES & +26 & 0,972 \\
\hline St Baudille & 38 & $\begin{array}{l}103 \mathrm{~A} \\
103 \mathrm{~A}\end{array}$ & $\begin{array}{l}= \\
=\end{array}$ & $\begin{array}{l}0,785 \\
0,668\end{array}$ & $\begin{array}{l}\text { MES } \\
\text { MES }\end{array}$ & $\begin{array}{l}+126 \\
0,923\end{array}$ & $\begin{array}{l}0,941 \\
0,969\end{array}$ \\
\hline
\end{tabular}




\section{Les expérimentations en cours}

\subsection{Mesures sur le site}

Les mesures comparatives en laboratoire avec le spectrophotomètre à fibre optique ayant donné satisfaction, nous avons installé l'appareillage sur le terrain dans les conditions de fonctionnement à terme.

L'appareillage se trouve dans une armoire thermostatée à l'aplomb du collecteur. La fibre optique plonge dans un bac primaire alimenté en eaux de ruissellement par une pompe immergée. Sur ce site la pompe est protégée par un dégrilleur automatique. L'appareillage est commandé par un microprocesseur qui assure les fonctions suivantes :

- mise en fonctionnement de la pompe immergée à partir d'une cote prédéterminée,

- déclenchement du préleveur échantillonneur d'eau automatique qui pompe dans le bac primaire,

- acquisition de la mesure toutes les secondes et intégration sur une minute,

- simulation de commande d'organes mobiles (vannes), - arrêt de la pompe et du préleveur en fin de ruissellement.

Dans le cadre de cette expérimentation nous ne testons pas de système de lavage automatique après une période de mesure. Ce dispositif sera peut être à mettre en œuvre si l'encrassement s'avère problématique.

\subsection{Développement d'un capteur ouvert}

L'appareillage testé jusqu'à présent nécessite obligatoirement un montage en dérivation qu'il faut alimenter par une pompe primaire immergée dans le collecteur d'assainissement. Les fibres otpiques pour cet appareil très simple sont courtes : 0,7 à $1,2 \mathrm{~m}$.

Un développement futur de ce type de mesure serait d'avoir recours à une fibre optique de grande longueur reliant directement le spectrophotomètre au collecteur. Il faudrait concevoir et construire une cellule ouverte qui serait positionnée en paroi du collecteur. Il convient alors de maîtriser les problèmes d'encrassement de la cellule de mesure et de protection de la fibre optique.

Une expérimentation dans ce sens est envisagée visant à concevoir et réaliser des cellules ouvertes, profilées et de chemin optique réglable permettant la mesure d'absorbance ou de l'intensité diffusée directement dans le collecteur.

\section{Conclusion}

La première phase de cette étude a permis de confirmer que dans le cadre des objectifs que nous nous étions fixés, la mesure de l'absorbance par spectrophotométrie est suffisamment corrélée avec la mesure de MES par la méthode AFNOR pour que l'on puisse apprécier la charge de l'eau par cette mesure optique.

Ces bons résultats doivent être confirmés par une multiplication des mesures sur différents types de collecteurs et par les résultats de l'expérimentation en cours sur le collecteur pluvial appelé "la Vieille Mer" dans des conditions de fonctionnement de terrain.

La conception et la construction d'une cellule de mesure qui pourrait être positionnée directement dans le collecteur permettrait alors de disposer d'un appareillage simple permettant une mesure instantanée du flux sur les sites pour lesquels des alternatives de gestion existent.

\section{Bibliographie}

Etude sur les turbidimètres.

Laboratoire régional de l'Ouest parisien. M.D. Grange, F. Pescheux.

Couplage d'un micro processeur à un capteur de qualité de l'eau par fibre optique, dans le cadre de la gestion d'un réseau d'assainissement.

Service départemental d'assainissement de la Seine-Saint-Denis. P. Chopard, R. Streiff.

Etude inter-agences appareils de mesure automatique des effluents. AFB Artois Picardie, octobre 1980.

Contribution au contrôle et à la régulation de la floculation des eaux usées.

Claude Casellas-Salha.

Utilisation de l'absorption ultra-violet à $254 \mathrm{~nm}$ pour la mesure en continu de la pollution organique dissoute. Analyse critique de l'analyseur OPM - Tinsley.

Université de Montpellier - Thèse de doctorat de $3^{\circ}$ cycle. R. Ben Aim, J.L. Marty, J. Coma, G. Parrain.

Les mesures optiques dans le contrôle des conditions de formation et de décantation des flocs physico-chimiques. Application aux eaux usées.

Université de Montpellier - Thèse de doctorat de $3^{\mathrm{e}}$ cycle. Maria Dolorès Rivas Vega. 